Fecha de recepción: abril 2020 Fecha de aceptación: mayo 2020 Versión final: junio 2020

\section{Complejidad e Incertidumbre: alternativas didácticas en prospectiva. El diseño desde el 2020.}

José María Aguirre ${ }^{(1)}$, Manuel Bazán ${ }^{(2)}$, Gerardo Castro ${ }^{(3)}$ y Enrique Goldes ${ }^{(4)}$

\begin{abstract}
Resumen: El aprendizaje del proceso de diseño, frente a un hecho tan complejo como la cultura, exige una posición teórica flexible y simultáneamente preparada para los inciertos cambios a los que el futuro nos enfrenta a diario. Los escenarios culturales del diseño son múltiples y, es en esa multiplicidad, donde radica el valor de su metodología: el proceso proyectual. Contextualizar, conceptualizar, producir, distribuir, comercializar, usar y desusar un producto/servicio de diseño requieren igualmente de usuarios particulares, contextos de interacción diferentes e interfases de contextualización específicas. Cada etapa es, al mismo tiempo, producto de la fase antecedente y proceso precedente del eslabón por venir. Pero, para el aprendizaje-enseñanza del diseño esto hoy resulta insuficiente. La definición disciplinar de Tomás Maldonado -de hace más de medio siglo- ha sido muy útil y, en algunas de sus aristas, aún vigente. Pero, resultó imprescindible, hace poco tiempo, una reformulación de la actual W.D.O. que podría quedar parcialmente obsoleta en virtud del contexto global presente y su proyección a un corto futuro.
\end{abstract}

Palabras clave: Complejidad - Incertidumbre - Estrategia - Prospectiva - Diseño.

[Resúmenes en inglés y portugués en la página 243]

(1) Arquitecto (Universidad Católica de Córdoba). Magíster en Docencia Universitaria (Universidad Tecnológica Nacional). Profesor de la Facultad de Arquitectura, Urbanismo y Diseño de la Universidad de Córdoba, Argentina (FAUD - UNC). Investigador categoría III SeCyT UNC y director de proyectos de investigación. Miembro del Consejo Asesor (Centro de Investigación en Diseño Industrial Córdoba, FAUD UNC). Representante por la FAUD UNC ante la Asociación Latinoamericana de Carreras de Diseño Industrial. Autor del libro DISEÑO. Aprendizaje, enseñanza y ego-axialidad. Ed. FAUD UNC, Córdoba.

(2) Diseñador Industrial (Universidad Nacional de Córdoba).Posgrado Vision in Products Design. Diseño Estratégico para el Futuro (Universidad Técnológica de Delft). Profesor (FADU / UNL y FAUD - UNC). Consultor Externo (Consultora Internacional Olivia y Consultora Internacional Round Feather). Diseño Estratégico. Design for Happiness Method.

(3) Diseñador Industrial (Universidad Nacional de Córdoba). Profesor (FAUD - UNC). Facilitador activo en proyectos de diseño, incubadora de empresas (UNC). 
(4) Diseñador Industrial (Universidad Nacional de Córdoba). Máster in Design Stratégico. Consorcio MIP Poli Design (Politecnico di Milano). Especialización en Gestión de Tecnologías Innovadoras (en curso). Profesor (FAUD - UNC). Responsable de proyectos de desarrollo para dependencias del estado municipal (ADEC). Facilitador en actividades de vinculación impulsadas por el estado provincial (MinCyT Córdoba).

\section{Introducción}

El aprendizaje del proceso de diseño, frente a un hecho tan complejo como la cultura, exige de una posición teórica flexible y simultáneamente preparada para los inciertos cambios a los que el futuro nos enfrenta a diario. Los escenarios culturales del diseño son múltiples $y$, es en esa multiplicidad, donde radica el valor de su metodología: el proceso proyectual. Contextualizar, conceptualizar, producir, distribuir, comercializar, usar y desusar un producto/servicio de diseño requieren igualmente de usuarios particulares, contextos de interacción diferentes e interfases de contextualización específicas. Cada etapa es, al mismo tiempo, producto de la fase antecedente y proceso precedente del eslabón por venir. Pero, para el aprendizaje-enseñanza del diseño esto hoy resulta insuficiente. La definición disciplinar de Tomás Maldonado -de hace más de medio siglo- ha sido muy útil y, en algunas de sus aristas, aún vigente. Pero, resultó imprescindible, hace poco tiempo, una reformulación de la actual World Design Organization (WDO) que podría quedar parcialmente obsoleta en virtud del contexto global presente y su proyección a un corto futuro.

Es en este marco general de tan grave y profunda incertidumbre, que nos preguntamos, como profesionales, docentes e investigadores del diseño, ¿cómo abordar su aprendizaje? ¿Y su enseñanza? La respuesta está, seguramente, en nuestra propia esencia, ya que son pocas las disciplinas que se encuentran tan preparadas a enfrentar la incertidumbre desde sus bases herramentales y metodológicas. Somos diseñadores, sí, pero recordemos que igualmente lo son nuestros estudiantes. La docencia debe dejar de mirarse endémicamente en sus abstractas programaciones y reorientar su búsqueda hacia los productos intelectuales de sus alumnos, con sus visiones en tiempo real de los infinitos micro contextos culturales en constante e incierto crecimiento. Allí está el futuro; ellos son el diseño desde el 2020.

\section{De nuestras bases disciplinares}

Tomamos como punto de partida disciplinar la última definición de Diseño Industrial, que en la 29a Asamblea General en Gwangju (Corea del Sur), el Comité de Práctica Profesional de la WDO (ex International Council of Societies of Industrial Design, ICSID) reveló como posición renovada frente al diseño industrial y que reza: 
Es innegable que el diseño industrial ha cambiado desde sus bases. La definición de fines de los sesenta del ICSID abrió el abanico de posibilidades y responsabilidades disciplinares de manera exhaustiva y resultó como útil guía durante décadas. Pero, desde hace un tiempo ya que, resulta insuficiente en algunos de sus conceptos. Hoy nos parece imposible mirar al hecho diseñado sin sus aristas experienciales o su dimensión de servicio, ambos aspectos que lo intrincan en la mayor de las complejidades culturales. Hoy el diseño ha perdido, además, el pudor de hablar de negocio y cliente en el mismo plano que se habla de valores sociales y ambientales. Pero es necesario permanecer atentos, porque el contexto que nos toca nos desafía nuevamente a repensar la disciplina en muy poco tiempo.

Este 2020 en recorrido nos ubica en una nueva bisagra histórica, no sólo al diseño, si no a la cultura toda, y la velocidad del cambio de dirección no deja tiempo para discusiones teóricas, tiempo que el diseño tuvo y gozó en los pasillos de la Bauhaus, el Vkhutemas, la HFG de Ulm o de nuestro Di Tella. Hoy la coyuntura es inédita y atraviesa a todos los factores culturales, en una trama que se re-estructura y re-formula casi a diario y que requiere de decisiones igualmente flexibles y preparadas para la permanente mutación de la realidad.

La universidad es parte fundamental de esa mutante realidad compleja y corresponsable de responder aceleradamente al desafío; nosotros, como parte de la universidad, no podemos escapar al conflicto y es igualmente importante asumir -como lo hiciera Gropius al finalizar la Primer Guerra- la enorme responsabilidad que el diseño tiene, tanto en la crisis actual, como en proponer caminos para una urgente salida de ella.

\section{De nuestras bases pedagógicas. De-construyendo el complejo aprendizaje- enseñanza}

Con la misma base de la propia redefinición disciplinar del WDO, es que afirmamos que el acto educativo es un proceso de múltiples fases, nunca un momento aislado del trabajo intelectual. Este proceso se monta sobre un plexo conceptual ya tradicional: la enseñanzaaprendizaje, pero es nuestra vocación deconstruirlo y reconfigurarlo como aprendizajeenseñanza, siendo primero y principal el acto constructor, y secundario y accesorio el acto propio de la guía docente. Nuestra función es accesoria en virtud de ser piezas absolutamente prescindibles e intercambiables de un mero sistema de referencias; en cambio la alumna, el alumno, son únicos. Poner el acento en el aprender por encima del enseñar, ubica igualmente en demanda de conocimiento tanto a alumnos como a docentes, en una verdadera práctica del conocimiento construido social y horizontalmente, y que necesariamente se enclava en la emoción propia de las relaciones interpersonales como motor. Deconstruir el tradicional proceso de enseñanza-aprendizaje nos reubica a los docentes en una posición de mayor privilegio aún, participando de una comunidad donde el número siempre nos favorece ¿Relación 50-1, en promedio? Cincuenta versiones de la realidad de 
las que podemos valernos, si didácticamente así lo planteamos. Pero, por lo general, el foco sigue en nosotros. No es el caso de nuestra Universidad Nacional de Córdoba (UNC), pero sabemos de instituciones en donde los espacios académicos (las cátedras) llevan como marca indeleble el apellido de sus responsables; qué mayor prueba que esa. Aunque esto no sea para nada nuevo.

Probablemente, en la historia se haya resaltado siempre y en mucha mayor medida a la figura genérica del maestro que la de sus alumnos, cuando fueron éstos los verdaderos motores de todo cambio. Es mucho más importante la impronta que diseñadores de la talla de Luciana González Franco, Sabrina Brugmann, Cristian Mohaded, Silvio Tinello, Gastón Bonaudi Pez, Manuel Torres o Francisco Gómez Paz están dejando en el territorio global del diseño que la que pueda dejar cualquiera de los que fuimos sus profesores. Tuvieron mayor trascendencia las obras de Marianne Brandt o Marcel Breuer que las de Walter Gropius, y las de éste y sus compañeros Mies Van der Roeh y Le Corbusier que las de Peter Beherens. Fue muchísimo mayor la huella de William Morris que la de su maestro en Oxford John Ruskin. Por qué razón entonces la docencia insiste endémicamente en regurgitar sus programaciones y currículum considerando al alumno como último depositario de tan magno saber, cuando la más auténtica pedagogía es aquella que recoge el capital cultural de los estudiantes, aprehende de ese capital lo nuevo o renovado que la cultura produce y ofrece a cambio herramientas metodológicas que permitan renovarlo aún más. Probablemente sea más difícil de implementar en niveles más tempranos de la educación pero, en la universidad, el conocimiento en reserva que pueden aportar los estudiantes es enorme y valiosísimo y es nuestra obligación y desafío el plantear estrategias didácticas que lo capitalicen y potencien. Resulta imperioso igualmente potenciar la voluntad de aprendizaje, y qué mejor manera que ofreciendo instrumentos para que los alumnos trabajen en el espacio educativo con el principal objetivo de revitalizar positivamente sus comunidades, comprometidos con los saberes propios, tanto en el saber como en el hacer.

\section{Educar para el conocimiento, educar para la emoción}

Piaget (1966), en su clásico libro Psicología de la inteligencia, define a ésta como a la equilibrada conjunción de factores cognitivos y afectivos. En muchas oportunidades -por no decir casi siempre- los criterios de evaluación que ponemos en juego los docentes en todo el sistema educativo, se orientan mucho más a evaluar el conocimiento que el compromiso emocional que nuestras alumnas y alumnos puedan establecer para con el contenido o los métodos puestos en juego (nunca mejor dicho) en nuestras aulas-talleres. De este modo, cerramos las opciones de articulación libre entre acomodación-asimilación, y las alumnas y alumnos reciben todo pre digerido. Los sujetos creativos necesitan de canales a través de los cuales expresar ese constante reflejo propositivo. Qué mejor que puedan expresarse como diseñadores en todas las áreas del conocimiento que representan la trama cultural. Cómo conseguir entonces el máximo compromiso afectivo-cognitivo: seguramente poniendo al estudiante en la posición de protagonista de su proceso de aprendizaje, y con la libertad de diseñar el cómo comunicar los resultados obtenidos. 
Este complejo asimilación-acomodación, que Piaget define como balance permanente en nuestra interacción con el medio, debiera ser la base que estructure los desafíos académicos a los que nuestros estudiantes se enfrentan a diario, buscando que todos se muevan libremente y con el herramental metodológico adecuado, en lo que nosotros llamamos esa zona de equilibrio normal (Aguirre, 2013). La z.e.n. es un territorio inteligente y alejado de los extremos básicos, como puede resultar, por un lado, el empirismo, fuertemente dependientes de lo que el medio manifiesta y, por el otro, el apriorismo, en fuerte sujeción con las capacidades innatas. Los estudiantes deben sentirse motivados a responder a las consignas de nuestras prácticas, pero pudiendo ejercer igualmente su capacidad de presionar al medio en su beneficio; después de todo, y si lleváramos el concepto a un nivel comunitario, eso no sería otra cosa que una alternativa definición del ejercicio del diseño. Si bien, para desempeñarse inteligentemente en el medio nuestros estudiantes necesitan de un buen bagaje de conocimientos conceptuales (de eso, nuestra enseñanza está al tope y por tradición ya centenaria), lo imperioso hoy es reforzar fuertemente la enseñanza en conocimientos metodológicos, que resultan en ese saber comodín que no depende de datos fijos y estrictos, no depende de una realidad única, si no que se abre a interactuar con cualquier colección de información, provenientes de múltiples realidades que son las que propiamente viven nuestros estudiantes; y a las múltiples realidades sólo se las aborda desde el conocimiento general ${ }^{1}$. No se trata de sumar prácticas, o peor, restarlas sólo por la cantidad de horas relativas que la materia en cuestión tenga en la curricula. Aunque suene obvio y poco se concreta, debemos articular y cualificar, entre las distintas áreas del saber, los métodos y contenidos que trabajamos con nuestros estudiantes. En palabras de Gombrich (1999 [1979]), estamos seguros que:

Debemos tratar de enseñar menos y no más, esto no nos absuelve de nuestra responsabilidad de ayudar al alumno a hacer un buen uso del tiempo así ganado. Por el contrario, debemos tratar con ahínco de encontrar alternativas válidas en vez de todavía más cursos y exámenes, a fin de facilitar al estudiante el mismo tipo de orientación, como mínimo, que la tradición del conocimiento general aportó a anteriores generaciones.

\section{Diseño centrado en la Vida}

En esta búsqueda del entendimiento de las nuevas problemáticas y alcances de la disciplina, a través de los diferentes proyectos de los alumnos ha surgido una conciencia colectiva sobre los límites del diseño, que nos permite cuestionar la realidad y establecer los grandes principios éticos que hacen innegociables el respeto y la conservación de la naturaleza, al tiempo que necesariamente deben garantizar nuevas políticas que regulen la proyectación y los sistemas de vida.

Morin (2004 [1990]) plantea, a través de su obra, que la óptica desde la complejidad obliga a considerar simultáneamente los fenómenos, los principios fundamentales que gobiernan esos fenómenos y los principios fundamentales metodológicos, lógicos y epistemo- 
lógicos que gobiernan y controlan el pensamiento. También nos dice que la complejidad se destaca principalmente por reconocer que, tanto en los fenómenos como en los conceptos, no sólo está presente lo preciso, lo matemático, lo físico, sino también la imprecisión, lo abstracto, la ambigüedad y la contradicción. El pensamiento complejo, en el cual se relacionan todos estos principios, hace que el pensamiento científico contemporáneo enfocado en la complejidad, sea más aproximado a la realidad. No nos sirve de nada enseñar desde el frío objetivo de la precisión, de la certeza, porque el contexto es ciertamente incierto. Más allá del juego de palabras, la incertidumbre es la clave para enfrentar lo complejo, para abordar el futuro. Pero aunque tengamos la gigantesca duda de cómo resolver el problema que nos ha ocasionado el desmesurado tamaño que ha adquirido hoy nuestra cultura material, tenemos la certeza absoluta ya de que la dificultad es real y de que no podemos seguir sometiendo al entorno a nuestros caprichos en pos de una especista búsqueda del bienestar.

De todas las formas de desarrollo e ideas de bienestar que pueden imaginarse para el futuro, la única que ya no se puede proponer es aquella que hasta hoy ha sido adoptada por los países industrializados y que, explícita o implícitamente, incluso los países menos industrializados han tomado como modelo de referencia. Un modelo de desarrollo y una idea de bienestar basados en una hipótesis muy simple y clara: para estar mejor hay que consumir más. Hoy sabemos que esta hipótesis, la relación directa entre bienestar y consumo, no siempre es verdadera. Sobre todo, sabemos que la promesa que propone no es sostenible, no puede, en otras palabras, hacerse extensiva por igual a todos los habitantes del Planeta (Manzini, 2013 [2004]).

Estas dos posturas nos arrojan luz sobre un futuro que asoma en principio como incierto, de la misma manera que lo ha hecho desde que el tiempo es tiempo. Lo novedoso hoy es que se suma a lo incierto lo insostenible, en una dimensión desconocida para nuestra historia como especie.

\section{Herramientas para una implementación como cátedra}

Nuestra preocupación como docentes es entonces la de brindar a nuestros estudiantes un espacio de aprendizaje para el cómo tratar las diferentes complejidades espaciales y temporales. El tratado de contextos complejos permite a los alumnos abordar y entender las causas que originan los problemas y cómo interactúan estos con otros, en relación con: un tiempo determinado o indeterminado; razones políticas detrás de los perfiles de gestión; aciertos o fracasos económicos; actividades sociales y conductas colectivas; expresiones regionales en el manejo de las diferentes artes, tanto las antiguas como las emergentes; por qué no se introducen o adoptan ciertas tecnologías, y cuáles podrían ser las adecuadas. Todos éstos son enlaces comunes en la puesta en escena de los proyectos de nuestros alumnos, y de este modo, profundizando en la problematización de la realidad, contextualizan 
los proyectos y diseñan propuestas de productos y servicios estratégicos hacia el futuro. En cada aula-taller se pone en juego la bajada desde la teoría, empleando alternativos enfoques metodológicos en el abordaje de estos proyectos, pero todo sobre la base de un meta-proyecto pedagógico que divide al año académico y de proyecto en dos grandes momentos: un momento de desarrollo extensivo y un momento de desarrollo intensivo. A cada uno de estos grandes momentos los dividimos a su vez en tres fases (las que conforman las seis etapas anuales: MDE: investigación, conceptualización, propuesta; MDI: diseño, ingeniería, y comunicación; donde la fase anterior siempre produce el contenido necesario para la fase subsiguiente y cuyo modelo procedimental sería: Input - Búsqueda - Output.

\section{CONTEXTO > Complejidad > Sostenibilidad > Innovación > Autonomía}

DESARROLLOEXTENSIVO

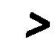

DESARROLLOINTENSIVO

Investigación > Conceptualización >

Propuesta

Diseño

$>$ Ingenieria

Comunicación

Aula 1. Design Thinking. Sin rotación de proyectos. Clase taller: Entendimiento, Discusión y Análisis Colectivo

Empatizar $>$ Definir $>$ Idear $>$ Prototipar $>$ Testear $>$ Presentar
Aula 2. Enginnering Design. Sin rotación de proyectos. Clase taller: Exposición, Crítica, Discusión y Ajustes

Identificación Identificación

de $>$ de Criterios y Posibles

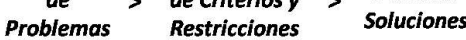

Construcción

la Propuesta del Prototipo > Presentación de

Testeo los Resultados

Aula 3. Strategic Design. Equipos con rotación de proyectos. Clase taller: Reunión de coordinadores (uno por equipo)

Oportunidad > Problema $>$ Concepto $>$ Producto $>$ Detalle > Comunicación

Fuente: Elaboración propia.

Como puede observarse en el cuadro anterior, en las aulas de Diseño Industrial III A entendimos que podíamos abordar la complejidad contextual desde la diversidad de metodologías y herramientas, partiendo desde saberes y prácticas naturalmente subjetivos. Esta diversidad nos ha permitido igualmente alinear momentos sincrónicos del proceso y la práctica, abriendo a la verificación de los niveles de conocimiento, entendimiento, 
aprendizaje, transferencia y autonomía que progresivamente van incorporando los estudiantes durante el año. Al mismo tiempo, podemos observar el grado de asimilación de la teoría y, con ello, la capacidad y habilidad de entender y discutir sobre la complejidad y el compromiso disciplinar frente a dicha realidad compleja.

Los resultados obtenidos desde el año 2011 muestran a las claras la madurez cultural y cívica de nuestros estudiantes en la observación de sus propias complejidades, y los resultados pueden observarse desde el principio del proceso, en la definición de las problemáticas abordadas desde la libertad de buscar y seguir las propias pistas que el medio les sugiere. Es así como nos encontramos con proyectos en tan variados nichos culturales como los de: agricultura sustentable; turismo; administración y uso de recursos hídricos en todas sus escalas; violencia institucional; seguridad en la vía pública; democratización de la alimentación y soberanía alimentaria; deportes de alto rendimiento; expresión y comunicación urbanas en ciudades inteligentes; violencia de género; tecnologías para la discapacidad; recuperación de ambientes naturales deprimidos o en riesgo; estimulación ante diferentes capacidades mentales; mecanismos sustentables para la construcción de viviendas; salud preventiva; mecanismos didácticos de sensibilización a edad temprana; y un larguísimo etcétera.

A medida que avanzan estos proyectos, frente a una incertidumbre que disminuye progresivamente, aumentan en paralelo las innovaciones y se comienzan a vislumbrar estrategias a corto, mediano y largo plazo. Los proyectos concluyen con el desarrollo de sistemas, productos y/o servicios, que buscan re insertarse en los escenarios tratados, para mejorarlos o cambiarlos de cara al bienestar futuro de todos sus actores. Desde la tesis doctoral Scenari, de Maschi (2002), en los albores de este siglo, el concepto de escenario cobró importancia en el universo intelectual del diseño, como lentes paradigmáticos que nos permiten desentrañar las diferentes situaciones culturales a las que el complejo sujeto-objeto se enfrenta permanentemente, con sus distintas calidades, actores y procesos implicados. En Latinoamérica, la definición de escenario trocó hasta engarzarse en propósitos más sociales. Seguramente ello obedece a que, en nuestro contexto, y a diferencia del europeo, no tenemos los presupuestos sociales mínimamente cubiertos, al punto de requerir una participación, inédita en la historia, de organizaciones no gubernamentales destinadas a responder con políticas concretas a todos esos presupuestos, cuando en verdad debiera ser el Estado, en sus diferentes escalas nacional, provincial y municipal, el responsable por cubrirlos adecuadamente.

En Argentina entonces, los escenarios de conceptualización, resolución o distribución, por nombrar sólo algunos, deben implicarse fuertemente en estructuras políticas en general muy deficientes, sin planes siquiera a mediano plazo y de alcances geográficamente muy modestos. Eso transforma al ejercicio del diseño en un verdadero desafío, cuasi ciclópeo. Sin embargo y como pudimos observar anteriormente, nuestros alumnos ponen mayoritariamente el foco sobre aquellas problemáticas fuertemente ligadas al tercer sector y virtualmente desatendidas por el estado. Podrá calificarse como riesgosa nuestra decisión de dejar en los estudiantes la libre elección de las problemáticas abordadas, y que casi todas ellas se mueven a las anchas en el territorio de irrealizables utopías. Convengamos en que, sin utopías el hombre nunca hubiera podido despegar sus pies del suelo más allá del impulso de sus propias piernas. El máximo riesgo que corren nuestros estudiantes es el 
mismo que nosotros como profesionales: el error. Pero nuestras expectativas de logro para con nuestros estudiantes a fin del ciclo nunca fue el hallazgo de la verdad del diseño. Si el resultado es fallido pero el proceso es exitoso, sería sólo nuestra la equivocación de evaluar como insuficientes a los productos finales de esos procesos. "El error quizá esté en pedirle la verdad a ese espejismo que es el hombre" (Roca, 2014).

\section{Cuatro ejes para la complejidad}

Reconocemos cuatro grandes ejes o escenarios que estructuran la trama cultural, que interactúan entre sí permanentemente, y que comprometen el abordaje del Diseño Complejo:

\section{El Contexto}

En términos generales es el conjunto de circunstancias que rodean a una situación, y sin las cuales no se puede comprender correctamente la trama cultural; hablamos de Complejidad y es fundamental, en este modelo conceptual de diseño, relevarlo por dos razones: la primera porque el contexto es en primera instancia $-y$ en palabras de Ortega \& Gasset (1914) - la circunstancia del hombre. Es la relación entre lo antrópico y el ambiente, ya sin muchas posibilidades de separar lo humano y su producto de lo natural, ya que son muchos los estudios que ponen en discusión esa falsa dicotomía (Castro y Zusman, 2009); y la segunda, para quitar del medio la idea de que contexto únicamente es el entorno natural. Como dijimos anteriormente, es todo aquello que hace demandas de la forma, que transforma todo lo que se ve y lo que no se ve. Habiendo desarrollado junto a nuestros alumnos este ejercicio durante muchos años ya, tenemos algunas certezas: las claves están en lo que se ve, pero éstas se complejizan en lo que no se ve. Es un error común simplificar o recortar el contexto con la idea de reducir el proyecto y hacerlo manejable. Esto puede llevar, aunque la práctica del oficio del diseño pudiera ser la correcta, a respuestas que provoquen grandes problemas a futuro, en esta frágil trama que intentamos re-construir. Si sabemos observarlo, el contexto nos mostrará claramente cuáles son los retos y los límites, así como también las redes que interactúan dentro de él, para poder así generar innovación y valor en el futuro.

El proceso de contextualización cultural es el primero de los eslabones de la cadena proyectual. El diseñador se ubica frente a la cultura y observa los síntomas que esta muestra, muchas veces de maneras no muy evidentes. Salvo aquellas culturas acostumbradas a convivir con la miseria y el desajuste, por lo general ninguna muestra a las claras cuáles son sus deficiencias. Sean o no evidentes, allí debe apuntar el diseñador verdaderamente comprometido con su contexto, más allá de los requerimientos del mercado, como ámbito parcial de la cultura. Si el mercado muestra desajustes, estos siempre estarán ligados a cuestiones financieras, lisa y llanamente a perder o ganar dinero. El diseño con compromiso social con su contexto cultural contemporáneo debe superar esos estrechos límites y desplegar sus herramientas en pro de corregir los desajustes propios del perder o ganar calidad de vida. En definitiva el diseño es un servicio, nunca un producto, y esta visión 
intangible de la cosa diseñada es la que actualmente está mostrando, y cada vez con más fuerza, la vanguardia artística y científica como camino a seguir en el futuro; el diseño debe mirar allí, si es que quiere sobrevivir como disciplina.

\section{Las Personas}

Como canales de vinculación con la Vida, las personas son para nosotros las protagonistas centrales en este modelo conceptual de diseño, y son las que aportan los datos más significativos, porque ellas poseen la clave de los hallazgos, de lo que debemos resolver, del porqué el mundo es como es, o simplemente cómo se ven afectados ellos por la hostilidad del mismo. Operar sobre lo cotidiano implica, pues, partir de la observación de las personas en su contexto, de sus preguntas y de sus modos de actuar, considerar las elecciones de las empresas y las políticas de los entes públicos a partir de la escala micro. En fin, para lo que concierne a las disciplinas del proyecto, significa referirse principalmente al campo de acción del diseño. Además podemos observar a través del estrecho lazo que desarrollan con productos y servicios, las interacciones y comportamientos que se establecen, y las dificultades que conllevan ${ }^{2}$. El contexto debiera ser una plataforma de la cual las personas pudieran apropiarse, para utilizarlo y sentirse cómodas desarrollando sus vidas, colectivamente e individualmente y en armonía con el entorno, y en donde los productos y servicios sean medios para alcanzar una vida mejor.

\section{Los productos y servicios}

Al trabajar, el hombre transforma su tiempo en potencial capacidad de adquirir productos y servicios útiles o inútiles, hermosos u horribles, agresivos, peligrosos, obscenos o sublimes, según una escala de valores que podemos sin duda racionalizar, pero que difícilmente podremos prever. En el interior de cada objeto conviven tensiones, que percibimos seductoras, repugnantes o indiferentes, de acuerdo a una química personal e inefable. Estas tensiones constituyen el ADN del objeto y son parte de sus cadenas de valor (o disvalor) y de sus ciclos de vida, pero también contribuyen a que el contexto sea más o menos hostil con nosotros.

\section{El tiempo}

Es una constante para el ser humano; no podemos dejarlo fuera del estudio, y además se torna fundamentalmente estratégico el incluirlo. Cuando hablamos de tiempo no podemos dejar de pensar en años, meses, semanas, días, horas, minutos y segundos, como escalas de tiempo-manejo convencionales. Ellos nos permiten establecer la métrica general del proyecto y, por ende, de las problemáticas. Así también trabajamos con lo que llamamos tiempos no convencionales; hablamos de décimas, centésimas y milésimas de segundo, como así también de decenios, centenios y milenios. Éstos son tiempo que escapan a la observación común; micro y macro momentos, que poseen datos reveladores, precisos y significativos para el abordaje de un proyecto innovativo. 
Como en todo tiempo histórico, existe en la actualidad una preocupación en el mundo científico por saber cuál forma tomará el mundo laboral en pocas décadas. Libros como Homo Deus (Harari, 2016), El diseño de los objetos del futuro (Norman, 2010), o la biblioteca Open Mind (Fundación BBVA, 2009-2020), dan prueba de los desafíos culturalmente radicales a los que el diseño puede enfrentarse mañana -pero no metafóricamente, sino literalmente mañana mismo-. Esos desafíos van montados sobre una creciente intangibilización de la cultura, una desmaterialización creciente de todas las interfases, y hasta una virtualización rayana en lo absurdo de las relaciones y vínculos sociales. Hoy, el contexto de pandemia que vivimos a nivel global, nos ubica frente a un futuro verdaderamente incierto y sin las herramientas para sortear los obstáculos que conlleva. Pero la coyuntura puede presentarse como un abismo o un trampolín. Podemos saltar a la próxima era como lo hiciera aquella humanidad a punto de extinguirse hace cien mil años, desde donde -para bien y para mal- no hemos parado de evolucionar como especie. Pero también podemos caer en un pozo del que resulte cada vez más difícil salir ¿Cómo prepararnos para un futuro cercano así de novedoso, si en nuestras universidades seguimos formando aún diseñadores enfocados en resolver un aislado producto material, sin advertir siquiera una mínima parte de las sinapsis infinitas que ese producto-servicio teje con la trama cultural? La respuesta es tan cruda como taxativa: como educadores, llegaremos tarde.

\section{A modo de conclusión}

La sociedad red nace como una nueva utopía, como modelo y proyecto al que tienden las sociedades del futuro. La adecuación a este modelo representa hoy en día uno de los desafíos principales de cualquier política educativa o cultural. Se trata de inducir, formar, adecuar la percepción y las actitudes intelectuales a las condiciones de saber de las nuevas sociedades. Todo nuestro sistema de conocimiento bascula en la dirección de un proceso fuertemente acelerado, capaz de suministrarnos un tipo de información que supere cualitativamente los modelos sobre los que se ha construido todo nuestro anterior sistema de aprendizaje y conocimiento (Jarauta, 2013).

En este Cuarto Nivel educativo que habitamos como cátedra, el diseño está dirigido por el contexto y centrado en vida de las personas, y utilizamos este método de búsqueda de la innovación y generación de valor, fundamentalmente descubriendo aquellos datos significativos y patrones claves que nos brinda el alrededor -particularmente, aquellos saberes en capital conocimiento de los propios estudiantes-, aprovechándolos como herramientas para re-diseñar el mundo en que vivimos. Por eso decimos que nuestra metodología está dirigida a la contextualización y centrada en el ser humano como ser natural, parte de una trama vital.

Diseñar es materializar procesos recursivos en donde funcionen estratégicamente las interdependencias entre factores, en pos de hacer que el mundo que habitamos sea coherente con el funcionamiento de la vida toda. Entonces y sólo entonces el proyecto impactará 
profundamente en el contexto, y la generación de valor de los productos y servicios estará concentrada en las interacciones entre éstos y las personas y según sus tiempos naturales, creando de esta manera entornos sostenibles y recursivos, en una nueva plataforma sociotécnica de cara a un futuro preferible.

\section{Notas}

1. Sobre el valor del conocimiento general resulta más que interesante revisar los escritos de Walter Gropius (1937), en cuanto a la necesidad de educar para la visión, y que en gran medida resulte en un debate que sigue ausente de los espacios educativos. Decía el alemán: Empero, debiera constituir nuestro más elevado objetivo producir ese tipo de hombres capaces de visualizar la totalidad, y no dejarse absorber demasiado pronto por los estrechos canales de la especialización. Nuestro siglo ha producido el tipo experto por millones; dejemos ahora lugar a los hombres de visión.

2. Cuanto nos recuerdan estos conceptos a la asignatura dictada por Paul Klee en el Vorkurs de la Bauhaus: el Dibujo para la Vida (Fundación Juan March, 2013).

\section{Listado de referencias bibliográficas}

$29^{\circ}$ General Assembly in Guangju, Professional Practice Committee (2015). Definition of Industrial Design. Obtenido de: www.wdo.org/about/definition/

Aguirre, J. M. (2013). Diseño. Enseñanza, aprendizaje \& ego-axialidad. Córdoba: Ed. FAUD UNC.

Biblioteca Open Mind (2009-2020). Ed. Fundación BBVA. Obtenida de: www.bbvaopen mind.com

Castro, H. y Zusman, P. (dic. 2009). En: Investigaciones geográficas $\mathrm{n}^{\circ}$ 70: Naturaleza y cultura: dualismo o hibridación. México: Ed. UNAM.

Fundación Juan March (2013). Paul Klee. Maestro de la Bauhaus. Madrid: Ed. Fund. Juan March.

Gombrich, E. (1999 [1979]). Ideales e ídolos. Madrid: Ed. Debate.

Gropius, W. (1963 [1937]). Alcances de la arquitectura integral. Buenos Aires: Ed. La Isla.

Harari, Y. N. (2016). Homo Deus. Buenos Aires: Ed. Debate.

Jarauta, F. (editor). En: Cuadernos de diseño 3. Diseño, innovación, empresa: Presentación.

Ed. IED Instituto Europeo de Diseño. Madrid 2013 [2009].

Manzini, E. (2013 [2004]). En: Cuadernos de Diseño 1: Pensar - proyectar el futuro. Ed. IED Instituto Europeo de Diseño. Madrid.

Maschi, S. (2002). Scenari. PhD thesis. Milano: Politecnico di Milano.

Morín, E. (2004 [1990]). Introducción al pensamiento complejo. Barcelona: Ed. Gedisa.

Norman, D. A. (2010). El diseño de los objetos del futuro. Madrid: Ed. Paidós. 
Ortega \& Gasset, J. (1914). Meditaciones del Quijote. Ed. Imprenta Clásica Española. Obtenido de: www.books.google.com.ar

Piaget, J. (1966). Psicología de la inteligencia. Buenos Aires: Ed. Psique.

Roca, J. M. (2014). Sobre el error: Fe de erratas. Belluno: Ed La Fábrica.

\begin{abstract}
Learning the design process, facing a fact as complex as culture, requires a flexible theoretical position and simultaneously prepared for the uncertain changes that the future faces us on a daily basis. The cultural scenarios of design are multiple and, it is in this multiplicity, where the value of its methodology lies: the design process. Contextualizing, conceptualizing, producing, distributing, marketing, using and unusing a design product / service also require particular users, different interaction contexts and specific contextualization interfaces. Each stage is, at the same time, the product of the antecedent phase and preceding process of the link to come. But, for the learning-teaching of design this is insufficient today. The disciplinary definition of Tomás Maldonado -of more than half a century ago- has been very useful and, in some of its edges, still in force. But, a reformulation of the current W.D.O. It could become partially obsolete due to the current global context and its projection in the short future.
\end{abstract}

Keywords: Complexity - Uncertainty - Strategy - Prospective - Design.

Resumo: Aprender o processo de design, enfrentando um fato tão complexo quanto a cultura, requer uma posição teórica flexível e simultaneamente preparada para as mudanças incertas que o futuro nos enfrenta diariamente. Os cenários culturais do design são múltiplos e é nessa multiplicidade que reside o valor de sua metodologia: o processo de design. Contextualizar, conceituar, produzir, distribuir, comercializar, usar e não usar um produto / serviço de design também requer usuários específicos, contextos de interação diferentes e interfaces de contextualização específicas. Cada estágio é, ao mesmo tempo, o produto da fase antecedente e do processo anterior do vínculo que está por vir. Mas, para o aprendizado-ensino do design, isso é insuficiente hoje. A definição disciplinar de Tomás Maldonado - de mais de meio século atrás- tem sido muito útil e, em algumas de suas arestas, ainda está em vigor. Mas, uma reformulação do atual W.D.O. Pode tornar-se parcialmente obsoleto devido ao atual contexto global e sua projeção no futuro próximo.

Palavras chave: Complexidade - Incerteza - Estratégia - Prospectiva - Design.

[Las traducciones de los abstracts fueron supervisadas por el autor de cada artículo] 